\title{
Performance Analysis of SACOCDMA-FSO System Using MD Codes
}

\author{
Bhanu Priya \\ Student, Department of Electronics and Communication Engineering \\ GNDU Regional Campus Jalandhar, Punjab, India \\ bpriya812@gmail.com
}

\begin{abstract}
Performance of SACOCDMA-FSO system using MD codes at 10 Gbps is evaluated. $M D$ code has become an area of interest in optical communication because of its ability to support many users at higher data rates, flexibility to select weight parameters. The proposed system was compared with SACOCDMA-FSO System employing RD codes in different conditions. The simulative results demonstrated that optical system based on MD codes is better than the system employing RD codes.
\end{abstract}

Keywords: Free space optics(FSO),Random Diagonal Codes (RD), Weather conditions, Transmit power levels, Bit Error Level, Transmission distance, Multi Diagonal Codes $(M D)$

\section{Introduction}

Free Space Optics has an edge over microwave and optical communication technology due to its high carrier frequency (of the order of THz) which allows it to absorb large number of users [1]. The underlying principle of Free Space Optical communication is a LASER driven technology in which at transmitter side LASER source along with telescope is used to transmit light through atmosphere and received by photo detector and telescope. Instead of transmitting light through silica fiber, light is made to propagate through air as it travels faster in atmosphere [2].FSO has been employed in various applications link i.e. building-building, aircraft-ground and satellite to ground links due to its enormous advantages like large capacity, quick installation, license-free long range spectrum at reduced cost [3].

In order to ensure enhanced security and asynchronous access OCDMA is first choice but it has major drawbacks i.e. MAI[4] caused due to other users and PIIN [5] which is similar to MAI and leads to expansion of spectrum beyond electrical bandwidth.SAC OCDMA System offers a better solution to minimize the effect of MAI and PIIN by employing fixed in phase cross-correlation codes [6].The Multi-Diagonal code is based on combination of diagonal matrix and has zero cross-correlation which nullifies the effect of MAI and PIIN. It provides maximum allowable BER at higher data rates with large number of users, ease of choosing the parameters i.e. code length, code weight, in-phase cross-correlation [7]. The combination of Multi-Diagonal code with Free Space optics is economical and has many advantages like enhanced security, minimum dispersion and narrow laser beam widths. In order to put these two ideas together many parameters are to be optimized such as transmitter and receiver apertures, diverging angle, attenuation (for clear, heavy rain, moderate fog and heavy haze) and operating wavelength.

The rest of the manuscript is organized as follows; Simulation setup of proposed system is described in next section. After it the results are discussed. Finally whole research paper is concluded in the end. 


\section{Simulation Setup}

Figure. 1 shows block diagram of proposed system with two users with help of MD codes. In this design continuous wave Laser is used at transmitter side according to the weight (i.e., 2 in this case).The data is first multiplexed from $\mathrm{CW}$ laser and then modulated by the use of Mach Zehnder modulator. After it multiplexer is used to collect the signal from different users. Free Space Optical channel with divergence angle of 0.25 mrad, transmitter aperture of $10 \mathrm{~cm}$ and receiver aperture of $20 \mathrm{~cm}$ is used. The data rate at which the whole design simulates is 10 Gbps. At the receiver side Demultiplexer is employed to distribute the signal to intended receiver so that no interference between multiple users occurs. After it Fiber Bragg Grating used selects a wavelength to be transmitted further. Photo-Detector converts optical signal into electrical one whereas filtering action is provided by Low Pass Filter. Finally the results are visualized by BER analyzer [8]. BER analyzer provides information regarding Eye-height, Quality factor, Threshold, Bit Error Rate. Moreover Optical spectrum visualizers can be used at any point of the design to visualize the spectrum and can conclude changes occurred in signal during transmission in optical domain.

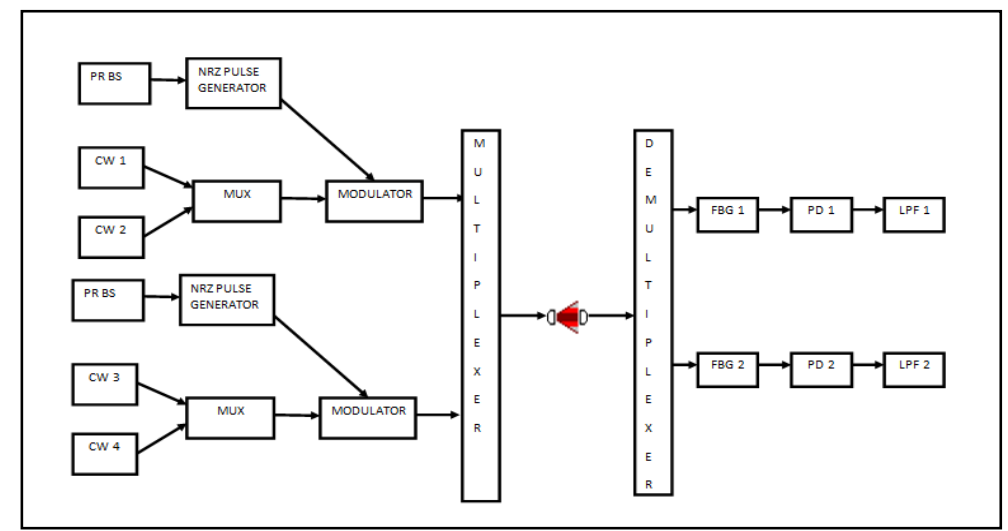

Figure 1. Block Diagram of SAC OCDMA-FSO System Using MD Codes

\section{Results and Discussion}

\subsection{Performance of SACOCDMA-FSO System Using MD Codes in Different Weather}

Simulations were carried out in OptiSystem version 11.0. Figure 2 shows BER performance of proposed system against transmission distance at $10 \mathrm{Gbps}$ and transmit power level of $0 \mathrm{dbm}$. It clearly explains the fact that BER increases with transmission distance in all weather conditions. It also clearly depicts that the proposed system performs well with clear weather and is unable to transmit the data at longer distances in heavy rain, moderate fog as the working of free space optical communication depends on the climatology and physical characteristics of location [9]. Eye diagram depicted in Figure. 3 clearly concludes that $\mathrm{h} 1>\mathrm{h} 2$ where $\mathrm{h} 1$ and $\mathrm{h} 2$ are eye height obtained with clear weather and heavy rain respectively, next to them is the eye diagram with moderate fog in which eye width is minimum which means the data is affected by intersymbol interference..All the parameters value used during simulation is arranged in Table 1 presented below. 
Table 1. List of Parameters Used During Simulation

\begin{tabular}{|c|c|}
\hline Parameters & Value \\
\hline $\begin{array}{c}\text { Operating wavelength } \\
\text { region }\end{array}$ & $1550 \mathrm{~nm}$ \\
\hline Signal Bit Rate & $10 \mathrm{Gbps}$ \\
\hline Transmitter aperture & $10 \mathrm{~cm}$ \\
\hline Receiver Aperture & $20 \mathrm{~cm}$ \\
\hline Beam Divergence & $0.25 \mathrm{mrad}$ \\
\hline Attenuation & $8.68 \mathrm{~dB} / \mathrm{km}$ \\
& $($ Heavy rain) \\
& $1 \mathrm{~dB} / \mathrm{km}(\mathrm{Clear}$ \\
weather $)$ \\
\\
\end{tabular}

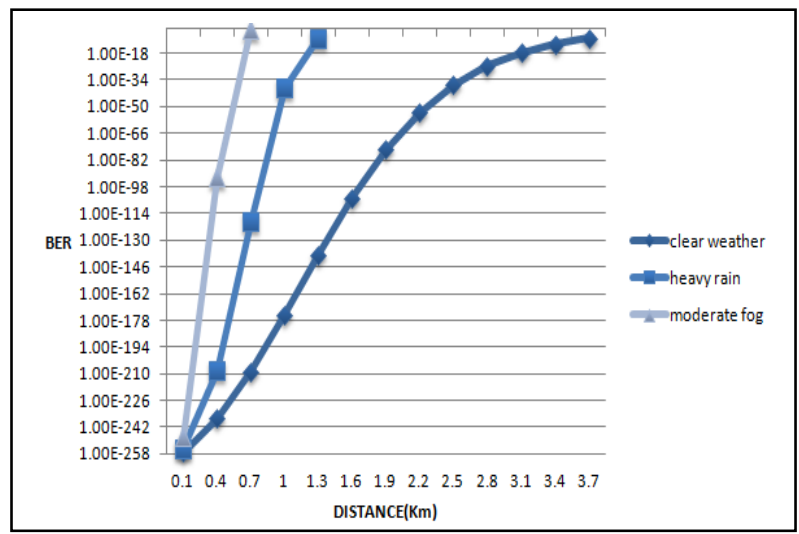

Figure 2. BER of SACOCDMA-FSO System Using MD Codes for Different Transmission Length in Different Weather Conditions

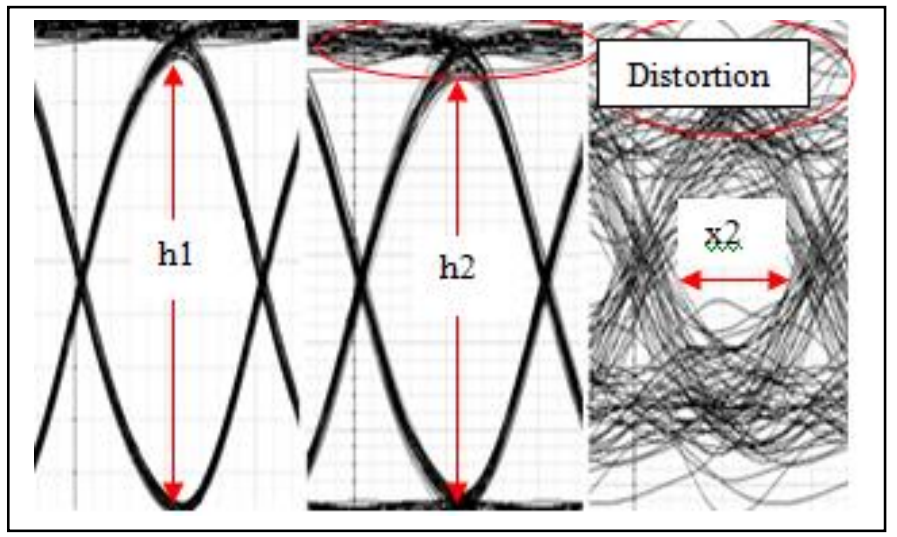

Figure 3. Eye Diagram of Proposed System with MD Codes at (a) Clear Weather (b) Heavy Rain (c) Moderate Fog 


\subsection{Comparison of Proposed System with MD and RD Codes in Heavy Rain}

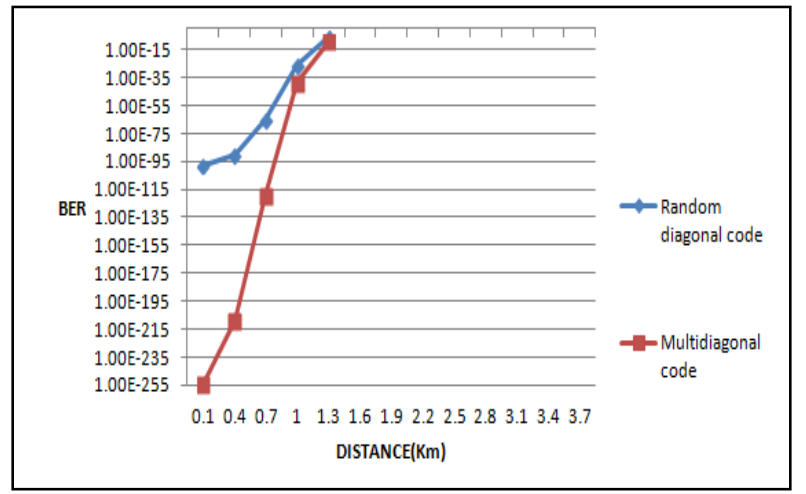

Figure 4. BER of Compared Systems against Different Transmission Length in Heavy Rain

Figure 4 depicts the comparison of proposed system with that of RD codes in heavy rain. It was found that SACOCDMA-FSO system using MD codes was able to cover 3.7 $\mathrm{km}, 1.3 \mathrm{~km}, 0.62 \mathrm{~km}$ in clear weather, heavy rain, moderate fog respectively whereas 3.4 $\mathrm{km}, 1.2 \mathrm{~km}, 0.59 \mathrm{~km}$ in clear weather, heavy rain, moderate fog respectively with help of $\mathrm{RD}$ codes. So the results show that the transmission distance improves by $8.8 \%, 8.3 \%$, and $5.08 \%$ in clear weather, heavy rain, and moderate fog respectively with help of MD codes [10].Figure. 5 presents the eye diagram of compared system at $1.2 \mathrm{~km}$ in heavy rain. The proposed system with RD provides BER of $\sim 10 \exp (-10)$ whereas with the help of MD BER of $\sim 10 \exp (-35)$ was achieved. From the eye diagram (Figure 5) it is very much clear that in case of MD code the eye height is open wide which means that there will be least chance of loss of data i.e. minimal intersymbol interference whereas in the eye diagram obtained with RD code suffers from jitter and the best time required to sample the data without error is minimum. The ability to withstand noise is less as the eye is not open wide.

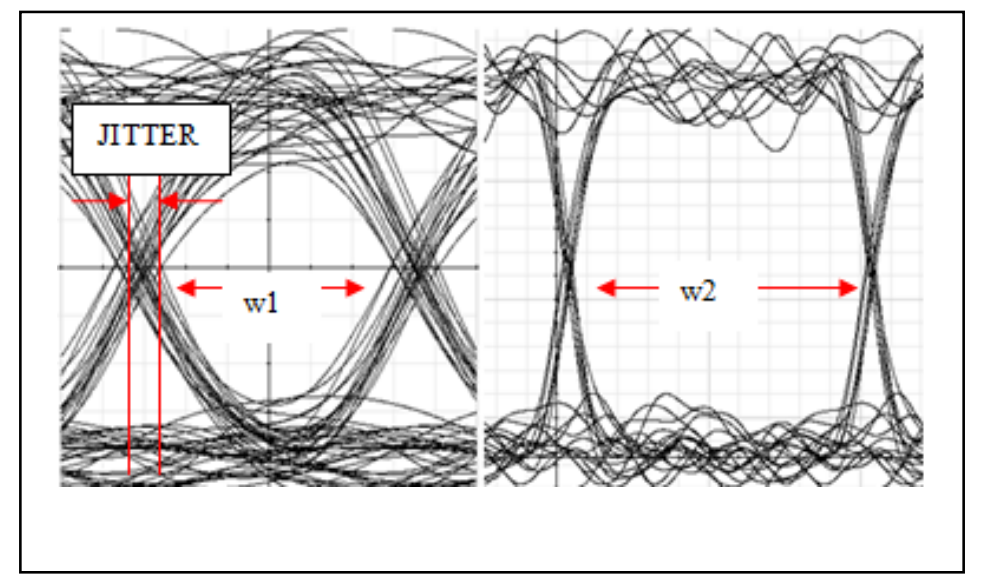

Figure 5. Eye Diagram of Proposed System with (a) RD Codes (b) MD Codes at $1.2 \mathrm{~km}$

\subsection{Comparison of Proposed System with MD and RD Codes at Different Power Levels}

Figure 6 presents the BER performance evaluated with respect to distance with different values of transmit power for both systems. In heavy rain at $-10 \mathrm{dbm}$ proposed system covers $0.65 \mathrm{~km}$, at $0 \mathrm{dbm}$ signal travels upto1.3km and at $5 \mathrm{dbm}$ it covers $1.7 \mathrm{~km}$. 
It can be concluded through graphic analysis and results mentioned above that performance of system is better when transmit power is larger $(5 \mathrm{dbm})$.As increasing transmit power levels increases SNR and helps the signal to withstand channel impairments i.e., attenuation, environmental conditions [11]. The data transmitted using $\mathrm{RD}$ codes undergoes distortion due to the minimum transmitted power (as shown in Figure.7).

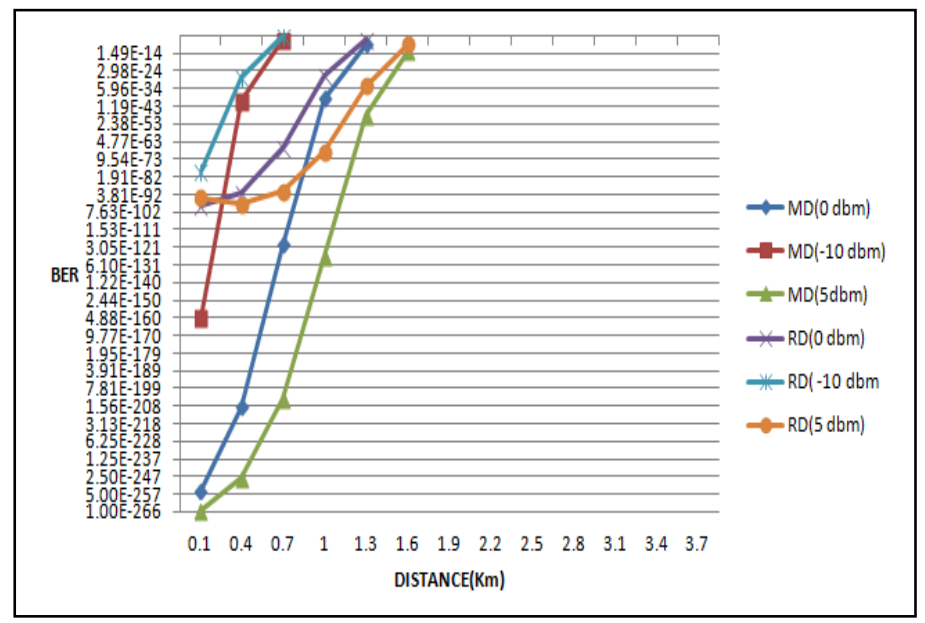

Figure 6. BER Performances of Both Systems against Distance at Different Values of Transmit Power

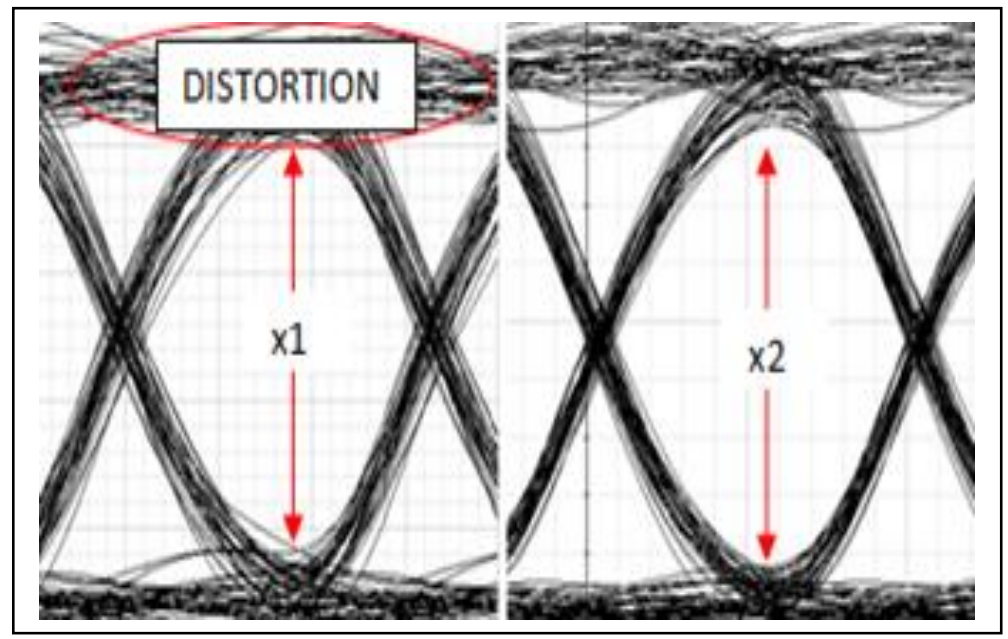

Figure 7. Eye Diagram of Proposed System with (a) RD Codes (b) MD Codes at $-10 \mathrm{dBm}$

\subsection{Comparison of Proposed System with MD and RD Codes at Different Data Rates}

Figure. 8 depicts the BER performance against transmission distance at different data rates i.e. $2.5 \mathrm{Gbps}$ and $10 \mathrm{Gbps}$ for both $\mathrm{MD}$ and $\mathrm{RD}$ codes. The graphic analysis shows that system functions well at lower data rates $(2.5 \mathrm{Gbps})$ because as data rates goes on increasing the pulse width decreases and it become difficult to differentiate between bit 1 and 0 which leads to dispersion [12-15].The Noise Margin in case of RD is less than MD code (Noise Margin gives the idea of immunity to noise) shown in Figure 9. 


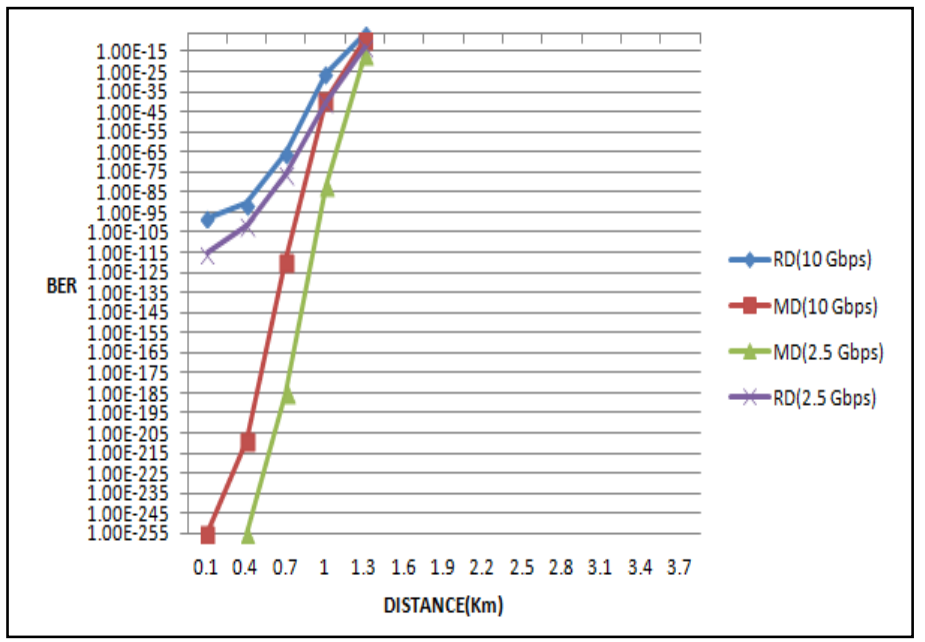

Figure 8. BER Performances of Both the Systems at Different Values of Data Rate

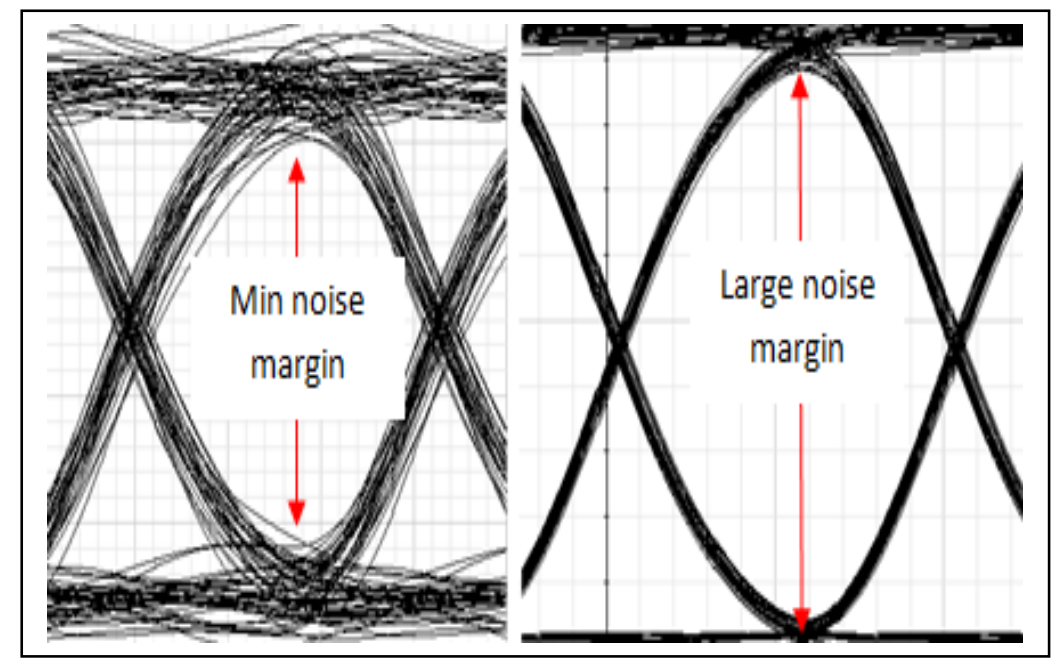

Figure 9. Eye Diagram of Proposed System with (a) RD Codes (b) MD Codes at $10 \mathrm{~Gb} / \mathrm{s}$

\section{Conclusion}

The paper successfully explored the performance of SACOCDMA-FSO system employing MD code and the comparative study of proposed system with that of RD code in different scenarios. It is perceptible that the proposed system presented in this paper is more efficient than the SACOCDMA-FSO system employing RD codes and the transmission distance improves by $8.8 \%, 8.3 \%$, and $5.08 \%$ in clear weather, heavy rain, and moderate fog respectively with the applications of MD codes.

\section{Acknowledgements}

I would like to acknowledge Guru Nanak Dev University for availing "Opt system 11.0" software. 


\section{References}

[1] H. Willebrand and B. S. Ghuman, "Free-Space Optics: Enabling Optical Connectivity in Today's Networks", Sams Publishing, Indianapolis, (2002).

[2] S. Arnon, J. Barry, G. Karagiannidis, R. Schober, and M. Uysal, Eds., "Advanced Optical Wireless Communication Systems", Cambridge University, (2012).

[3] T. H. Carbonneau and D. R. Wisley, "Opportunities and challenges for optical wireless: the competitive advantage of free space telecommunications link in today's crowded marketplace". Wireless technologies and systems: millimeter wave and optical, Proceedings of SPIE Research no. 3232, pp. 119128, (1997).

[4] S. Tseng and J. S. We, "A new code family suitable for high-rate SACOCDMA PONs applications", IEEE J. Select. Area Commun, Research no. 28, no. 6, (2010).

[5] E. D. J. Smith, R. J. Blaikie and D. P. Taylor, "Performance enhancement of spectral amplitude-coding optical CDMA using pulse-position modulation", IEEE Trans.Commun. Research no. 46, (1998), pp. $1176-1184$

[6] A. M. Safar, S. A. Aljunid, A. Razif, A. J. Abdullah, J. M. Nordin and M. N. M. Saad, "Enhancement of Zero Cross Correlation Code for Optical CDMA Network System", International Conference on Communications and Information Technology, ( 2012).

[7] T. H. Abd, S. Aljunid, H. A. Fadhil, I. F. Radhi, R. Ahmad, and M. Rashid, "Performance improvement of hybrid SCM SAC-OCDMA networks using multi-diagonal code," Sci. Res. Essays, vol. 7, pp. 12621272, (2012).

[8] M. Noshad and K. Jamshidi, "Code family for modified spectral amplitude-coding OCDMA system and performance analysis", Opt., Commun. Network, pp. 344-354, (2010).

[9] Z. Wang, A. Chowdhury, P. R. Prucnal, "Optical CDMA code wavelength conversion using PPLN to improve transmission security”, IEEE Photon. Technol, vol. 21, pp. 383-385, (2009).

[10] M. Noshad, K. Jamshidi, "Novel codes family for modified spectral-amplitude coding OCDMA systems and performance analysis", IEEE/OSA J, Opt. Commun. Network, (2010), pp. 344-354, vol. 2.

[11] S. Khaleghi, M. R. Pakravan, "Quality of service provisioning in optical CDMA packet networks”, Opt. Commun. Network, vol. 2, (2010), pp. 283-292.

[12] R. K. Z. Sahbudin, M. Kamarulzaman, S. Hitam, M. Mokhtar and S. B. A. Anas, "Performance of SAC OCDMA-FSO communication systems", Optik, (2013), pp. 2870, vol. 124.

[13] R. K. Z. Sahbudin, M. K. Abdullah, M. Mokhtar, S. Hitam and S. B. A. Anas, "Design andcost performance of decoding technique for hybrid subcarrier spectral amplitude coding-optical code division multiple access system", Comput. Sci., pp. 1525-1531, vol. 7, no. 10, (2011).

[14] M. K. Abdullah, S. A. Aljunid, S. B. A. Anas, R. K. Z. Sahbudin, M. Mokhtar, "A new optical spectral amplitude coding sequence", Khazani-Syed (KS) Code, in: Inter-national Conference on Information and Communication Technology, pp. 266-278, (2007).

[15] R. K. Z. Sahbudin, M. K. Abdullah, M. Mokhtar, S. B. A. Anas and S. Hitam, "Performance of subcarrier OCDMA system with complementary subtraction detection technique", World Acad. Sci. Eng. Technol., (2011), pp. 1421-1426.

\section{Authors}

Bhanu Priya, received the degree of B.TECH (ECE) from Punjab Technical University and is currently pursuing MTECH (ECE) from Guru Nanak Dev University . Her research interest are OCDMA, optical communication, FSO, digital signal processing . 
International Journal of Hybrid Information Technology

Vol.8, No. 5 (2015) 